\title{
Gender-focused Social Innovation and the Role of International Development Volunteers in Promoting Women's Economic Empowerment
}

\author{
Rebecca Tiessen, Tiffany Laursen, Benjamin J. Lough, with Tabitha Mirza
}

\section{Introduction}

Social innovation programming is an essential component of international development and economic empowerment projects and is linked to economic growth and improved job opportunities (Pandey/Kumar 2019). Integral to social innovation and economic expansion, women are increasingly targeted as an "untapped driver of growth" (Devex 2013; Collins 2016). However, despite a growing number of commitments to invest in women's economic empowerment, women are still often excluded from entrepreneurial investments, receive less funding for innovative business ventures, and are less likely to be engaged in full-time jobs compared to men (Lough/Wells, forthcoming; World Bank 2014; World Bank 2021). Furthermore, only 1 in 3 small, medium, and large businesses globally are owned by women, and this rate drops to 1 in 4 in lower-income countries (Halim 2020). Evidence of gender inequality in the economic sector can also be found in women's disproportionate time spent on unpaid care and household labor (2.5 times more than men), occupational segregation, lack of maternal leave provisions, and overall disparity in earnings between women and men (International Labour Organization 2017; Ferrant/Pesando/Nowacka 2014; United Nations 2015).

Addressing the economic insecurity of women and girls is central to several global strategies, including the Sustainable Development Goals (SDGs). These goals focus on providing equal value for unpaid care work and promoting shared domestic responsibilities, as well as ensuring full participation in leadership and decision-making by women. However, the economic empowerment of women also requires simultaneous commitments to addressing the social and systemic practices that perpetuate gender inequality by reducing inequitable access to economic resources and financial services while opening new economic activities and supports to scale up social innovation.

Gender-focused social innovation (GFSI) marks a departure from previous development strategies aimed at women's economic empowerment in several ways. First, GFSI moves away from essentialist notions of economic empowerment that assume women's incorporation in the market as business actors will eliminate gender inequality (Collins 2016). Second, bringing a transnational feminist lens, a GFSI approach to development programming rejects neoliberal discourses 
constructing subaltern women's work as that which falls outside the purview of traditional market activities that are considered to be 'productive' (Collins 2016). GFSI calls attention to the exploitation of gendered and unpaid labor upon which economies - local and global in scale - are dependent. Moreover, GFSI perspectives shift from narrow definitions of empowerment that instrumentalize gender in economic development projects and move toward a view of improved gender relations as a trigger, process, and product of social transformation. In this way, GFSI moves away from traditional market interventions and enhances our knowledge of innovations that are difficult to measure (Collins 2016; Blake/Hanson 2005).

As an example of GFSI in practice, process innovations may emerge from the interactions between marginalized and privileged groups who seek to combat cultural norms that perpetuate cycles of gendered economic exclusion (Pandey/Kumar 2019; Blake/Hanson 2005). In this way, GFSI acknowledges the gendered relations of power that underlie systemic inequalities and seeks to disrupt them by enhancing peoples' capacity to act. Consistent with this approach, this paper considers diverse strategies for the promotion of GFSI and women's economic empowerment across ten countries. We draw on examples of the programmatic commitments and the characteristics of program support, namely, the role of international development volunteers (IDVs) in advancing gender equality in economic empowerment and social innovation initiatives.

Several processes and outcomes, as well as challenges and opportunities for social innovation, emerge as they relate to the capacity building efforts of IDVs in relation to GFSI. An important starting point for evaluating GFSI and the contributions of transnational actors is to consider the aspirational nature of GFSI, its process orientation, and the significance of a GFSI approach to all stages of the development project, including decision-making, project planning, implementation, and monitoring and development of activities with diverse groups of project participants (Eriksson 2014; da Silva Filho/de Souza/de Souza Lessa 2018). Within GFSI initiatives, we underscore the role of IDVs in facilitating economic change beyond instrumentalist women's economic empowerment programs by considering strategies that disrupt social inequalities through these transnational interventions. The findings from this study document how IDVs facilitate women's economic empowerment as both a process and product of GFSI by challenging systemic social inequalities and sustainable development in partner communities.

\section{Literature Review: Social Innovation and Gender Empowerment}

Innovation is conceptualized as the development of new or improved products, services, and processes (Baregheh/Rowley/Sambrook 2009). Innovation in the economic sector facilitates financial growth and is a measure of economic and positive 
social change (Eriksson 2014). As a social response to complex and deepening economic, political, and environmental crises (Hillier/MacCallum/Moulaert 2013), social innovation (SI) is defined in relation to the fulfillment of "human development ambitions" (Neumeier 2012: 49). Characteristics of social innovation include participatory, collaborative, and capacity-building dimensions of change that support the needs and priorities of marginalized groups that offer novel solutions to social inequality (Hillier/MacCallum/Moulaert 2013). Social innovation is also characterized by commitments to local ownership, context specificity, and inclusion and ownership of the process by those who are meant to benefit from the interventions (da Silva Filho/de Souza/de Souza Lessa 2018; Cunha/Benneworth/ Oliveira 2015; Hillier/MacCallum/Moulaert 2013; Correia/Gomez/Oliveira 2016).

Gender equitable processes drive innovation and contribute to innovative products (Eriksonn 2014). Strategies that aim to include women in social innovations can range from short-term or limited techniques such as targeting women and girls to facilitate their inclusion within existing systems, to longer-term and transformational approaches that change the methods of inequality to facilitate new opportunities for women's and girls' participation (Rao/Tiessen 2020; Alsos/Hytti/ Ljunnggren 2013). However, not all of these strategies are wholly productive; several limitations of women's economic empowerment are captured by scholars who examine persistent inequalities within existing neoliberal economic processes (Hillier/MacCallum/Moulaert 2013; Morton/Muchiri/Swiss 2020). Moreover, a neoliberalist approach that sincerely links the achievement of "gender equality to economic growth" increases the risk of reproducing the "very structural issues that feminists, and gender and development, seek to trouble and transform" (Morton/Muchiri/Swiss 2020: 333).

An alternative to neoliberal forms of women's economic empowerment is outlined in GFSI, which considers angles, structures, and assumptions and how these are components of social transformation and elements of both process and outputs of innovation (Eriksson 2014; Hillier/MacCallum/Moulaert 2013; Correia/Gomez/ Oliveira 2016). Through the GFSI lens, gender inclusion and improved social relations can transform inequitable processes that prevent marginalized groups from active participation in their own development and are focused on power and agency (Rao/Tiessen 2020; Hillier/MacCallum/Moulaert 2013). GFSI therefore recognizes and re-generates the "power marginalized people already possess" (Morton/ Muchiri/Swiss 2020:333). It does not overlook, or further neglect people based on "class, regional, sex, ethnic, and socio-economic differences" (Rao/Tiessen 2020: 355; Eriksson 2014). In this way, GFSI can be used as a tool to achieve intersectional and transformative development through changes to the decision-making processes, the implementation of plans, and the monitoring and development of 
activities for enhanced fairness and equity among diverse groups (Eriksson 2014; da Silva Filho/de Souza/de Souza Lessa 2018).

International strategies designed to promote GFSI, and women's economic empowerment often include a range of actors, including transnational actors, aid workers, and international volunteers. While recognizing the value contributed by the wide range of these transnational actors, the research in this study considers the role and impact of IDVs in the promotion of GFSI programming engaged in timely opportunities to rethink, develop, and standardize the core organizational processes through a gender-aware lens (Lough/Tiessen 2019; Eriksson 2014).

Social innovation programs that are predominantly focused on the promotion of gender equality and women's empowerment are an essential advancement in international development solutions. As such, they shine a spotlight on the specific gender inequalities and women-specific needs of development programming. As described by a collaboration of scholars of the gendered nature of social innovation: "Gendered social innovation encompasses the identification of unsolved societal challenges of gender inequality and unmet needs among women or men as underrepresented or disadvantaged groups in various areas, motivating the development of new solutions by inclusive innovation processes" (Lindberg/Forsberg/ Karlberg 2015: 472).

A feminist theoretical lens through which we can examine GFSI emphasizes the role that context (place and people) plays in producing the conditions that enable or negate gendered social innovations, the nature of the social innovations commonly adopted, the process of delivering programming, and the ongoing needs and opportunities for gender-sensitive social innovations. Embracing a feminist lens considers the social and economic environment and its role in valuing and promoting certain kinds of innovative activity as well as devaluing or discouraging other forms of innovation. Furthermore, the social identities of innovators affect, and are affected by, the social and economic environment, contributing to ways that innovations get defined and promoted. A gender-focussed lens in social innovation provides insights into social and geographic contexts, as well as the enabling or limiting characteristics of those contexts (Blake/Hanson 2005). Thus, feminist and gender-focused priorities in social innovation shape, and are simultaneously shaped by, the negotiations over the understanding of contextually specific feminist approaches. (Lindberg et al. 2015)

An analysis of gender relations filtered through the lens of feminist social innovation is also shaped by the kind of social innovations introduced, including the nature of the relationship taking place in transnational spaces, such as the role and contributions of transnational actors. Scholarship in GFSI has, therefore, reinforced some of the core elements or drivers of social innovation, namely being 
inclusive, exchanging ideas and values, expanding and changing relationships and roles played by program participants, and diverse forms of support from donors. Applying a gender lens to these drivers allows us to consider the following questions: how to be inclusive in line with gender inequalities (context and people); how to ensure that a diversity of voices are represented in the exchange of ideas; how to deepen our consideration of the gendered roles and gender relationships that exist; how these relationships can change to facilitate social innovation from diverse people and in various contexts; and how to provide diverse forms of transnational support that will enable a gender and feminist lens in social innovation programming. Answers to these questions can enable a transformational approach to transnational feminist scholarship on social innovation.

A transformational and transnational feminist approach to social innovation considers gender-specific dimensions in a comprehensive way, focusing not just on the needs and interests of women and girls but also on the societal and structural barriers to their social innovation opportunities and the pathways to achieving gender equality. In other words, a feminist approach allows us to understand the power dynamics that perpetuate inequality of opportunity or relational considerations that might promote more gender equality by breaking down the nature and impact of 'place and people'. The emphasis on the context of place (i.e., community and culture-specific analysis) and people (i.e., transnational actors in their relation to local community members) is central to the analysis in this paper.

In addition to paying attention to context, a feminist approach considers opportunities for an intersectional lens to analyze the overlapping factors that can contribute to failure, success, or barriers to achieving social innovations. These intersectional realities include a variety of factors in addition to gender such as race, age, (dis)ability, ethnicity, class, etc. (see Lough/Wells, forthcoming).

In sum, transnational feminism offers a useful theoretical lens for breaking down the role and impact of place and people in our analysis of gender-focused social innovations. We employ transnational feminism in this paper because it helps us understand the opportunities and barriers of GFSI, the nature of GFSI interventions and how they support or counter feminist values, and the role of transnational actors working in local spaces. Specifically, transnational feminism underscores the global and cross-national impacts of social, historical, political, and economic activities carried out by diverse actors navigating their relationships to achieve social goals. In this way, the study provides new insight into contextually-specific feminist interpretations and perspectives of social innovation programming. 


\section{Methods}

Interviews were conducted in 2018 - 2019 with 150 partner organization staff in ten countries. Once all interviews were transcribed, data were coded to identify common themes throughout. Data were analyzed using discourse analysis. The introduction to this special edition has more detailed information on the methodology for data collection and the analysis of findings.

\section{Findings}

Findings from the data highlight the social innovation processes and results facilitated by IDVs working in community-based organizations. These findings provide insights into the nature and extent of IDVs' support to gender equality and women's empowerment (GEWE), and social innovation programming. IDVs were widely recognized for introducing new ideas in support of social innovation and building the capacity of local communities, including supporting organization staff with skills and competencies needed to advance social innovation programs and to support entrepreneurship training. The contributions made by IDVs were significant for social innovations in numerous development sectors, from agricultural programming to microfinance and tourism. However, despite many successes and opportunities created through the work of the IDVs, several challenges were also highlighted, particularly in relation to IDVs' understanding of cultural context and their local interpretations of how to achieve gender equality. Respondents that participated in the research believed that improved knowledge of local culture and enhanced skills among IDVs were needed to navigate different cultural contexts. They asserted that heightened cultural knowledge and competence were central needs in the delivery of future transnational support by IDVs working in international development programs.

In the sections that follow, we consider the nature and extent to which feminism is seen as a guiding lens for GFSI programs that have clear goals of women's economic empowerment across all ten countries. We outline how feminism is interpreted differently and situated within national and local realities and cultural practices. This is important for thinking about GFSI programs through a feminist lens because these findings reinforce the need to design GEWE programming within local contexts, keeping people and place at the forefront of development interventions.

\subsection{New Insights for Innovative Programming in Place}

Partner organization staff valued IDVs for their fresh perspectives, new ideas, and contributions to knowledge. As a participant from Tanzania noted: "What I value the most is [their] experience and perception of things". This participant went on 
to explain how the different backgrounds and perspectives allow for "fresh eyes on some old stuff," which enables organization staff to modify or improve programming. The new perspectives generated through interactions with IDVs are seen, however, in a generative way: "These experiences we are sharing from their country and our country, our perception of things, it develops both of us." This is an important insight as it shows the significance of shared and mutual learning in transnational spaces.

Among the shared knowledge and mutual contributions are new ideas in support of local innovations. Partner organization staff highlighted several examples of innovations shared by IDVs. These interventions were considered to have, as one participant from Tanzania explained, a "huge economic and social impact". This example highlighted the innovation shared by IDVs for biogas generation using waste to generate electricity. The community benefited from this project because it resulted in monetary savings. This intervention also facilitated training and knowledge building for all community members and established a protocol to ensure that young mothers are able to benefit from the training. Specifically, the project incorporated childcare support for mothers who wanted to access the training.

One example of a program in Ghana included creating a childcare facility and providing childcare services for mothers who wanted to participate in the trainings. This initiative was proposed by an IDV and was considered "quite unique" by local actors. This project ultimately led to a much more inclusive training program for a diverse set of community members. The inclusivity protocols included in this initiative were highly valued by partner organizations who saw impacts in terms of education, training, and knowledge sharing for those who were previously prevented from such opportunities. As one of the Ghanaian participants said: "I value most their contribution" to these new ideas and approaches - contributions that "are not measurable."

The second example of IDV support for innovative programming in Ghana included help with the design of a monitoring tool that could capture and measure gender inclusion in radio programming. A participant from Ghana added: "There was nothing like monitoring the gender responsiveness of a radio program. But with the help of the tools, we were able to give feedback to the radio team on how gender responsive their program was." The information generated from this evaluation tool allowed the radio program team to keep track of the number of women who called, the number of women interviewed, and the number of women who contributed to the program. The results of this initiative led to better equity in radio program representation with efforts to always have one man and one woman hosting a radio program together. Combined with this initiative was a training session that helped the staff members understand how gender inequality permeated organizational practice and helped the participants to "understand the way we are 
continuing the whole stereo-typing information dissemination that goes on focusing only on men."

Several programs implemented in Nepal tracked the impacts on women's participation in economic opportunities. Outcomes of these programs include socio-economic development, including the establishment of "114 sub-sector producers' groups (PGs) in vegetable farming (mainly cauliflower and tomatoes), milk production, and goat-raising in the three project districts" (CECI 2018). The programs focused on including women and marginalized groups (Dalits in particular) with a goal of maximizing benefits through project interventions. These efforts resulted in a high degree of participation among marginalized groups including a participation rate of $72 \%$ women, $65 \%$ youth, and representation of Dalits and single women (CECI 2018). The project focussed on skills development, management of agricultural practices, and livestock activities. Interview participants in Nepal highlighted a number of ways that IDVs contributed to these programs, including economic empowerment programs designed to reduce the financial dependence of women on men. Specific projects promoting economic empowerment could be found in commercial agricultural projects, women's leadership and ownership of projects, and the creation of opportunities for women to enhance skills in the economic sector, promoting women entrepreneurs and cooperative management.

The primary goal of the Nepal program was to create opportunities for women and youth to benefit from technical training to facilitate better inclusion of women in the economy, on economic decision-making bodies, and in political spaces. IDVs provide this support in partnership with the locally-based organization staff. All 15 of the interview participants in Nepal provided examples of the role that IDVs play in supporting gender-focus social innovation and entrepreneurship programming, including activities such as the development of a guiding manual for a cooperative, trainings, video production, and proposal writing, among other activities. In one example, the partner organization staff explained how the IDV wrote a project proposal that received funding to start up an essential oil business that benefited more than 75 women in the community through a new business venture. In addition to the proposal writing, the IDV also helped with the business development and marketing of the product.

Other IDVs in Nepal played a pivotal role in helping local organization staff translate their goals and priorities into actions. For example, IDVs were able to help the organization's staff develop a "concrete plan about how and where to begin" with gender equality programming and policies. The IDVs helped develop planning strategies (two-year action plans). Specific targets of these initiatives included increasing the number of women members in the cooperatives and including women in the policy and decision-making processes. As one participant for Nepal noted: "Only after the arrival of ... volunteers, we realized the need for and importance of GESI 
[gender equality and social inclusion] in the organization.” This example reinforces the importance of transnational actors in providing knowledge and ideas for translating ideas into practice. The priority of a stronger focus on women's needs and gender equality was already established by the organization in Nepal, and the volunteer's role was to help translate that idea into a reality.

Participants from Nepal provided several additional examples of how the volunteers used their knowledge of gender equality and social inclusion to improve development programming. As one interviewee from Nepal explained, the volunteers conducted research on farmers who were involved in the milk production sector over a long period of time: their problems, quality production, and maintenance of those milk products, which further helped in developing the new proposals and launching the new programs. The IDVs actively engaged the local farmers in these communities and ensured they were involved in the milk production. Over, time the IDVs realized that the milk was not being consumed by other communities and the IDVs saw this as an opportunity to expand the business and gain access to local markets where the farmers could sell the milk. Cultural marginalization of these farmers had previously made it difficult for them to access markets. Other communities in the area saw the possibilities created for the milk farmers who would bring the milk to the market to sell and began bringing their own goods to the market, including meat from buffaloes and cows. The IDVs worked with those excluded communities to help them understand the steps needed to bring their goods to market and encouraged them to develop their businesses in new ways. The knowledge of one of the IDVs who grew up on a cattle farm in Canada was particularly welcomed. The IDV had advanced knowledge about cattle, their digestive systems, and how best to feed and care for the cows. In addition to this practical knowledge, IDVs played an advocacy role for women farmers who began conversations with communities and partner organizations about the nature and extent of women's involvement in the dairy sector. However, as the IDVs noted, only men have legal control over the agricultural business. Through these observations, partner organizations began to think of new training programs to support women's rights, empowerment, and legal changes to support women's ownership of businesses.

Similar examples were provided in Tanzania, where partner organization staff explained the valuable contributions of IDVs who were able to identify knowledge gaps or missed opportunities. The IDVs were able to share these observations with partner organizations, and together, new initiatives and training opportunities were created to bridge knowledge gaps. The projects in Tanzania focused heavily on fruits and vegetable markets and business growth, and ecotourism. To support the fruits and vegetable agricultural sector, programs were geared to increasing entrepreneurial opportunities, business, and agronomic skills among women and 
youth and to improving productivity and market information and linkages to markets for women and youth.

In Tanzania, partner organizations provided several examples of the role of IDVs in promoting economic empowerment and gender equality in the areas of fruit production and processing, ecotourism, and agricultural programming. One example included a poultry production opportunity that was introduced to Maasai women since women often do not reap the benefits of raising livestock. The opportunity to raise chickens gave women a new source of income and an economic opportunity that they could own and control. This project also included gender sensitivity training for men and women in the community to ensure that women's labour is linked to financial gains from these projects and also developing greater economic independence and decision-making power.

Examples from Tanzania also included a significant emphasis on the importance of gender equality training and knowledge sharing. In some cases, partner organization staff considered this understanding of gender equality as a core feature of a feminist approach to development. In other cases, some participants explained they were not familiar with the meaning of feminism or did not consider it a helpful guiding concept for the work they were doing.

In Malawi, IDVs were able to build organizational capacity by introducing new reporting templates that would allow the organization to capture data better. As a participant from Malawi explained, IDVs worked with office staff to develop a tool that tracks: "the contributions per member, per month, per group so that we are able to track...strides we are making. If we have a group, if the savings are not increasing, the group is dead." The small contributions made by IDVs were often explained in relation to their ability to integrate a helpful innovation - such as the tracking tool - into an organization's projects. They also remarked on the IDVs' ability to provide innovative suggestions to improve existing initiatives in new ways.

One partner organization in Vietnam explained the contribution of an IDV who integrated principles of corporate social responsibility (CSR) to the organization. The staff member explained how the IDV shared information about the need for the industry to "pay back to the communities." This knowledge helped the organizations in Vietnam think about fairness in corporations that extended well beyond gender equality to strengthen overall economic fairness.

Another IDV working in Vietnam filled important gaps in organizational assessments of fairness by identifying new priorities for the organizations and creating an opportunity to critically reflect on these priorities using a SWOT (strengths, weaknesses, opportunities, and threats) analysis. Through the SWOT analysis, organizations were able to consider a large number of variables needed for the 
equitable delivery of their programs and the actions needed to achieve their next steps. The recommendations that arose from this process allowed the organizations to consider their place in the broader organizational community, how they can be more competitive, and ways to communicate their ideas better. In just one year, the organization noted a large number of changes.

GFSI programs in Guatemala aimed to impact economic, social, and professional outcomes for more than 12,000 women and youth. These projects were designed to strengthen an inclusive market, increase economic competitiveness, and build the capacities of community members through technical support. Some projects focused on the agricultural sector designed to provide sustainable growth and to foster inclusive market systems. Other projects focused on enhancing the participation of women and youth in the agricultural industry through the creation of new opportunities in the coffee industry (production, management, administration, and commercialization). Similarly, the cardamom export sector has many opportunities for job creation for women and youth. Another project area included the food processing industry with a focus on job creation for women and youth, specifically in processing and packaging fruits and vegetables and in the preparation of marmalades and jellies. These projects resulted in increased incomes (by 25\%) for agricultural producers, 40,000 trees planted, support to 1,332 coffee producers $-25 \%$ of whom were women - and collective outcomes that led to improved price negotiation skills, production infrastructures, technological innovation, processing, and improved governance and women's participation (CECI no date).

The support provided by IDVs in Guatemala led to entrepreneurial development, including the creation of 769 small and micro businesses and 1,539 jobs, with priority given to women and youth. Business plans were created by " 907 micro-entrepreneurs and business service centers developed gender equity policies and implemented special projects for women and youth." (CECI no date). The IDVs were integral to the delivery of these programs, providing a range of resources and support through new ideas, networking, skills development, and capacity building. As one partner organization in Guatemala noted, the IDV "was here for one year, and she helped us on the commercial side of things. It was a pilot project I was doing ... it was the first time they gave us the opportunity to develop business opportunities for our members, and [the volunteer sending organization's] primary interest was to help smaller producers in the field."

The research conducted in Guatemala highlighted that the majority of IDVs engaged in GFSI programs focused on women's economic empowerment. IDVs provided support to: develop business opportunities and building partnerships with female-led businesses, enhance an organization's social programs that target both male and female youth, and conduct a human resources evaluation with a particular focus on gender relations within the organization's workplace. Specifically, 
IDVs partnered with local organizations and community members to increase women's salaries by building a business development model with a local business that was widely celebrated in the community. Other IDVs offered workshops on how to start a business with a focus on the empowerment of women to take advantage of opportunities. IDVs also focused on finding new ways of managing and handling the finances of the project to ensure funds distributed were used for their intended purposes. Finally, IDVs generated business opportunities for community members in order to help smaller producers achieve economic empowerment. Overall, the Guatemala interview participants agreed (8/15 strongly agreed and $7 / 15$ somewhat agreed) that the IDVs contributed to improving the partner organization's capacity to address gender equality.

Across these different interpretations and implementations of GEWE programming in place, the interview participants noted a high level of satisfaction with the work that IDVs do to promote gender equality. The contributions of IDVs to GEWE within specific contexts was found to be significant. Understanding the local cultural contexts were central features of partner organization's reflections on the role and impact of IDVs. Within these specific locations, interviewees indicated that IDVs help to build local capacity, address and break down barriers to promote GEWE and advocate for policy enhancements while engaging with local knowledge holders and compromising on approaches to fit within local contexts. The partner organizations attributed IDVs with bringing in fresh perspectives, curiosity and energy, commitment to empowerment, capacity building, skills development, and changes that resulted from new attitudes and behaviors to organizations and communities. As transnational actors working in place, IDVs play an important role in shaping new perspectives, attitudes, and reciprocal learning that are only available through the engagement of external actors. Participants also described indirect contributions to GEWE that transformed local attitudes towards gender norms and expectations through IDVs' lived examples and informal dialogue with community members. Further, IDVs were also able to provide support to local staff in the day-to-day running of business operations through communication and IT skills, allowing for the strengthening of the host partner organization's efforts towards GEWE.

\subsection{IDV Considerations for Improved GFSI Outcomes}

Though IDVs were highly praised for their effectiveness in promoting GFSI in partner countries, respondents also noted several challenges that limited IDVs' ability to fully impart sustainable change in their respective communities. Specifically, their level of expertise, limited time commitments, and language barriers were frequently identified as significant factors that impeded GFSI outcomes in social innovation programs. Respondents expressed their frustrations with volunteers' 
expertise and professional experience with GFSI, as many volunteers lacked formal training in gender equality work; however, it is important to note that while many IDVs lacked professional experience in gender equality, most were viewed as flexible and willing to learn to assist with GFSI initiatives.

Finally, respondents agreed that limited time commitments impacted the effectiveness of IDVs' integration into the community and, thus, their impacts on GEWE initiatives as well. They described a lack of continuity after volunteers leave because their organizations do not have enough personnel to fill the full-time positions required to sustain the projects that were started by volunteers. Some of the partner organization staff also highlighted the need for IDVs to spend more time learning about the local context and drawing fewer conclusions before they had sufficient knowledge. A participant from Peru noted: "to transform things; you need to understand and not judge." Similarly, in Senegal, a staff member said she expected more understanding from the IDVs and to see differences in how countries run their enterprises, since "from the logistic and operational points of view, it is not the same".

\section{Discussion and Analysis}

Partner organizations working to promote social innovation in GEWE in the Global South engage in a unique relationship and exchange focused on the differences in cultural gender norms within the Global North and South. This exchange is characterized by an embrace of gender equality perspectives that have emerged both locally and internationally. At times, these interactions resulted in clashes related to cultural norms creating tension, confusion, but oftentimes, innovative initiatives. Partner organizations often promoted (and implemented) core programming geared to improving gender relations and women's empowerment while also adapting what may be culturally irrelevant or unacceptable. Our findings reinforce the significance of context in relation to the interpretation of GFSI. This helps us understand and expand transnational feminist theory by advancing an intersectional approach and pursuing a broader strategy that more fully incorporates community-centered inclusive practices. A social-inclusion approach to GEWE (particularly evident in Nepal) offers an important insight into transnational feminism by highlighting the way that partner organizations navigate donor demands in partnership with IDVs as transnational actors working to advance GFSI programming.

The examples provided in the findings above highlight the specific contributions made by IDVs to promote GFSI and women's economic empowerment. The nature of the gender-focused innovations introduced by the IDVs opened up spaces for women's participation and addressed some of the systemic barriers to women's inclusion. Other examples included IDVs playing a role in providing resources 
or supports that could facilitate women's participation. In so doing, the projects moved from an approach of targeting women's participation in economic empowerment programs to eliminating some of the barriers to their participation by identifying the factors that prevent participation and finding ways (through social innovations) to circumvent or tackle those barriers.

Social innovations are important for enhancing women's participation and addressing the systemic barriers to participation. On the other hand, several important limitations were also noted as areas to be addressed to enhance the contributions of IDVs. The implications for improving empowerment programming emphasize how social innovations introduced by IDVs are best proposed through participatory and informed processes that involve IDVs asking critical questions and engaging partners in discussions about what is possible before making suggestions or imposing changes that may not be culturally appropriate or sensitive.

Taken together, findings from this study shine a light on the critical importance of people and place as a central lens for the implementation and analysis of GEWE programming where transnational volunteers work in people-to-people relationships with local actors. Policies that aim to advance gender equality cannot be regulated or administered in a vacuum. To work effectively, the gender equality policies that drive programming must consider the specific social, historical, political, economic, religious, and other conditions that define people's lived reality in particular contexts. While transnational actors often work to advance gender equality through standard methods such as recognizing the value of unpaid care work, promoting shared domestic responsibilities, ensuring women's full participation in leadership and decision-making, and empowering women economically, they must carefully "situate" these approaches within the milieu of place and human relationships. Furthermore, by situating interventions in context, IDVs can also deliver capacity building methods that enable partner organizations to "formulate and own" their own approach to internal partner organization transformation so that gender-focused innovation outcomes can answer the needs of the local context" long after the IDVs' mandate is over (Rao/Tiessen 2020: 365; Devereux 2008; Hillier/MacCallum/Moulaert 2013; da Silva Filho/de Souza/de Souza Lessa 2018; Lough/Tiessen 2020).

\section{Conclusion}

The findings examined here lend themselves to several key recommendations. The first recommendation is to ensure that IDVs have the necessary knowledge and training in gender equality and women's empowerment when tasked with working on GFSI projects. While technical training is an important first step, it must also be paired with contextual information and knowledge about local cultural norms and practices as well as culturally distinct approaches to addressing gender 
equality. International volunteer cooperation organizations (IVCOs), universities and colleges, faith-based organizations, and other institutions that send volunteers abroad need to structure their programs to enable IDVs to spend time listening and learning from partners about their own gender equality strategies and feminist practices. The pathways toward achieving gender equality differ across countries, and organizations and volunteers need to adjust strategies to each place. By tailoring their implementation strategies, volunteers can ensure that their interventions are culturally appropriate, while also helping to build social cohesion and gaining community buy-in.

IDVs play a valuable role in advancing gender equality programming, and they contribute to new perspectives in gender-focused social innovations. Likewise, community members and partner organization staff who are embedded in local places can contribute their own education, experience, knowledge, and training to enhance the capacities of international volunteers. To do feminist work effectively, IDVs and the organizations that facilitate their placements must promote the exchange of ideas, engage in reciprocal learning opportunities, work to understand and apply emic feminist frameworks that work effectively in place, and stay fully open to culturally-divergent pathways for achieving gender equality.

Social inclusion is not a magic bullet to promote GEWE but rather a strategy to further explore its potential boundaries, limitations, and promises. Ultimately, the findings in this study remind us that the implementation of feminist principles and GFSI hinges on the depth of understanding that transnational actors have about the cultural norms (context) and the spaces for integrating a gendered-focused approach that is culturally specific and valued. A transnational feminist approach to GFSI is linked to effective program implementation because GFSI programming requires a commitment to understanding local context and values, including a respect for the resilience and social transformation of local communities. Successful strategies focused on gender equality and social inclusion must begin with a context-specific feminist approach while also maintaining social cohesion, preventing conflict, and creating buy-in among staff and community members to cooperatively implement and sustain GFSI projects.

\section{Bibliography}

Alsos, Gry Agnete; Hytti, Ulla; Elisabet, Ljunggren (2013): Gender and innovation. State of the art and a research agenda. In: International Journal of Gender and Entrepreneurship, vol. 5, no. 3, pp. 236-256.

Baregheh, Anahita; Rowley, Jennifer; Sambrook, Sally (2009): Towards a multidisciplinary definition of innovation. In: Management Decision, vol. 47, no. 8, pp. 1323-1339.

Blake, Megan K; Hanson, Susan (2005): Rethinking innovation. Context and gender. In: Environment and Planning A, vol. 37, pp. 681-701. 
CECI (2018): Annual Report 2017-2018. https://www.ceci.ca/data/ceci-nepal-annualreport-2017-18.pdf (28.10.2021).

CECl (N.D.): Prosol. https://ceci.ca/en/projects/prosol (6.17.2021).

Collins, Andrea M. (2016): "Empowerment" as efficiency and participation. Gender in responsible agricultural investment principles. In: International Feminist Journal of Politics, vol. 18 , no. 4. pp. 559-573.

Correia, Suzanne Érica Nobrega; Gomez, Carla Regina Pasa; Oliveira, Veronica Macario De (2016): Dimensions of social innovation and the roles of organizational actor: the proposition of a framework. In: RAM. Revista de Administração Mackenzie, vol. 17, no. 6, pp. 102-133.

da Silva Filho, José Carlos Lázaro; Ana Clara Aparecida Alves, de Souza, Bruno, de Souza Lessa (2018): Social innovation and the promotion of local economic development. In: Innovation \& Management Review, vol. 16, no. 1, pp. 1-17.

Devereux, Peter (2008): International volunteering for development and sustainability. Outdated paternalism or a radical response to globalisation? In: Development in Practice, vol. 18 , no. 3, pp. 357-370.

Devex (2013): Women entrepreneurs: An untapped driver of economic growth. https://www. devex.com/news/sponsored/women-entrepreneurs-an-untapped-driver-of-economic-growth-81458 (6.29.2021).

Eriksson, Anna Fogelberg (2014): A gender perspective as trigger and facilitator of innovation. In: International Journal of Gender and Entrepreneurship, vol. 6, no. 2, pp. 163-180.

Ferrant, Gaëlle; Pesando, Luca Maria; Nowacka, Keiko (2014): Unpaid care work: The missing link in the analysis of gender gaps in labour outcomes. https://www.oecd.org/dev/development-gender/Unpaid_care_work.pdf (6.29.2021).

Halim, Daniel (2020): Women Entrepreneurs Needed-Stat! https://blogs.worldbank.org/opendata/women-entrepreneurs-needed-stat (28.10.2021).

Hillier, Jean; MacCallum, Diana; Moulaert, Frank (2013): Social innovation: Intuition, precept, concept, theory, and practice. In: ResearchGate, vol. 14, no. 41, pp.13-24.

International Labour Organization (2017): World Employment Social Outlook: Trends for Women 2017. http://www.ilo.org/wcmsp5/groups/public/---dgreports/---inst/documents/ publication/Wcms_557245.pdf (6.29.2021).

Lindberg, Malin; Forsberg, Lena; Karlberg, Helena (2015): Gendered social innovation - a theoretical lens for analysing structural transformation in organizations and society. In: International Journal of Social Entrepreneurship and Innovation, vol. 3, no. 6, pp. 472-483.

Lough, Benjamin J.; Tiessen, Rebecca (2019): International volunteering capacity development. Volunteer partner organization experiences of mitigating factors for effective practice. In: Forum for Development Studies, vol. 46, no. 2, pp. 299-320.

Lough, Benjamin J.; S, Wells (forthcoming): Intersectional analysis of gender, race, and geography on the funds raised by social ventures.

Cunha, Jorge; Benneworth, Paul; Oliveira, Pedro (2015): Social entrepreneurship and social innovation. A conceptual distinction. In: Carmo Farinha; Luís M.; Ferreira J. J. M., Smith, H. L.,; Bagchi-Sen S (ed.): Handbook of research on global competitive advantage through innovation and entrepreneurship. Pennsylvania, pp. 616-639. 
Morton, Sam E; Muchiri, Judyannet; Swiss, Liam (2020): Which feminism(s)? For whom? Intersectionality in Canada's Feminist International Assistance Policy. In: International Journal: Canada's Journal of Global Policy Analysis, vol. 75, no. 3, pp. 329-348.

Neumeier, Stefan (2012): Why do social innovations in rural development matter and should they be considered more seriously in rural development research? - Proposal for a stronger focus on social innovations in rural development research. In: Sociologia ruralis, vol. 52, no. 1, pp. 48-69.

Pandey, Umesh Chandra; Chhabi, Kumar (2019): SDG5 - Gender Equality and Empowerment of Women and Girls. Bingley.

Rao, Sheila; Tiessen, Rebecca (2020): Whose feminism(s)? Overseas partner organizations' perceptions of Canada's Feminist International Assistance Policy, In: International Journal: Canada's Journal of Global Policy Analysis, vol. 75, no. 3, pp. 349-366.

World Bank (2014): Gender at Work: A Companion to the World Development Report on Jobs. https://documents.worldbank.org/en/publication/documents-reports/documentdetail/884131468332686103/gender-at-work-a-companion-to-the-world-development-reporton-jobs (17.10.2021).

World Bank (2021): Labor force participation rate, female (\% of female population ages 15) (modeled ILO estimate). https://data.worldbank.org/indicator/sl.tlf.cact.fe.zs (6.29.2021).

United Nations (2015): The World's Women 2015.https://unstats.un.org/unsd/gender/downloads/worldswomen2015_report.pdf (28.10.2021). 